\title{
Can Current Algebra Determine the Low Energy $\pi \pi$ Scattering Amplitude?
}

\author{
J. Itiopoulos \\ CERN - Geneva
}

(Nuovo Cimento. $53 \mathrm{~A}, 552(1968)$ )

Page 555, second line after Table I, instead of:

"Furthermore, we must notice that the solution must, in any case, be disregarded... ". Should read:

«Furthermore, we must notice that the solution (b) must, in any case, bo disregarded...". 